\title{
CRISPR/Cas9-mediated knockout of clinically relevant alloantigenes in human primary $\mathrm{T}$ cells
}

\author{
Elahe Kamali ${ }^{1}$, Fatemeh Rahbarizadeh ${ }^{2 *}$, Zohreh Hojati ${ }^{1 *}$ and Morten Frödin ${ }^{3}$
}

\begin{abstract}
Background: The ability of CRISPR/Cas9 to mutate any desired genomic locus is being increasingly explored in the emerging area of cancer immunotherapy. In this respect, current efforts are mostly focused on the use of autologous (i.e. patient-derived) T cells. The autologous approach, however, has drawbacks in terms of manufacturing time, cost, feasibility and scalability that can affect therapeutic outcome or wider clinical application. The use of allogeneic T cells from healthy donors may overcome these limitations. For this strategy to work, the endogenous T cell receptor (TCR) needs to be knocked out in order to reduce off-tumor, graft-versus-host-disease (GvHD). Furthermore, CD52 may be knocked out in the donor T cells, since this leaves them resistant to the commonly used anti-CD52 monoclonal antibody lymphodepletion regimen aiming to suppress rejection of the infused T cells by the recipient. Despite the great prospect, genetic manipulation of human T cells remains challenging, in particular how to deliver the engineering reagents: virus-mediated delivery entails the inherent risk of altering cancer gene expression by the genomically integrated CRISPR/Cas9. This is avoided by delivery of CRIS PR/Cas9 as ribonucleoproteins, which, however, are fragile and technically demanding to produce. Electroporation of CRISPR/Cas9 expression plasmids would bypass the above issues, as this approach is simple, the reagents are robust and easily produced and delivery is transient.
\end{abstract}

Results: Here, we tested knockout of either TCR or CD52 in human primary T cells, using electroporation of CRISPR/ Cas9 plasmids. After validating the CRISPR/Cas9 constructs in human 293 T cells by Tracking of Indels by Decomposition (TIDE) and Indel Detection by Amplicon Analysis (IDAA) on-target genomic analysis, we evaluated their efficacy in primary $T$ cells. Four days after electroporation with the constructs, genomic analysis revealed a knockout rate of $12-14 \%$ for the two genes, which translated into $7-8 \%$ of cells showing complete loss of surface expression of TCR and CD52 proteins, as determined by flow cytometry analysis.

Conclusion: Our results demonstrate that genomic knockout by electroporation of plasmids encoding CRISPR/Cas9 is technically feasible in human primary $T$ cells, albeit at low efficiency.

Keywords: CRISPR/Cas9, Knockout, Genome editing, TCR, T cell

\footnotetext{
* Correspondence: z.hojati@sci.ui.ac.ir; rahbarif@modares.ac.ir

'Department of Cell and Molecular Biology \& Microbiology, Faculty of Biological Science and Technology, University of Isfahan, Isfahan, Iran

${ }^{2}$ Department of Medical Biotechnology, Faculty of Medical Sciences, Tarbiat Modares University, Tehran, Iran

Full list of author information is available at the end of the article
}

(C) The Author(s). 2021 Open Access This article is licensed under a Creative Commons Attribution 4.0 International License, which permits use, sharing, adaptation, distribution and reproduction in any medium or format, as long as you give appropriate credit to the original author(s) and the source, provide a link to the Creative Commons licence, and indicate if changes were made. The images or other third party material in this article are included in the article's Creative Commons licence, unless indicated otherwise in a credit line to the material. If material is not included in the article's Creative Commons licence and your intended use is not permitted by statutory regulation or exceeds the permitted use, you will need to obtain permission directly from the copyright holder. To view a copy of this licence, visit http://creativecommons.org/licenses/by/4.0/. The Creative Commons Public Domain Dedication waiver (http://creativecommons.org/publicdomain/zero/1.0/) applies to the data made available in this article, unless otherwise stated in a credit line to the data. 


\section{Background}

The development of genome editing technologies has enabled site-specific gene disruption or modification at unprecedented efficiencies and holds the promise of treating a broad range of diseases at the genetic level or through engineered cells $[1,2]$. Four categories of genome editing systems have been developed: meganucleases, zinc-finger nucleases (ZFNs), transcription activator-like effector nucleases (TALENs), and clustered regularly interspaced short palindromic repeats (CRIS PR)/CRISPR-associated (Cas) nucleases [3-6]. These have a common mode of action by binding to a userdefined sequence in genomic DNA and inducing a targeted DNA double-strand break (DSB) [7]. If the DSB occurs in a coding sequence, gene knockout can be achieved by the action of either of two cellular DNA repair pathways; the canonical non-homologous end joining (cNHEJ) pathway and the alternative NHEJ (altNHEJ, also known as microhomology-mediated endjoining, MMEJ) pathway $[8,9]$. Both pathways typically generate small nucleotide insertion or deletion (indel) mutations at the cleavage site and if the indel disrupts the reading frame, a premature stop codon and functional gene knockout will result. The extent to which either of these repair pathways are utilized depends on the nature of the DSB, the sequence flanking the DSB, the cell type, and the cell cycle stage, where the DSB happens [10-13].

Challenging de novo engineering has impeded widespread exploitation of meganucleases, ZFNs and TALE Ns [14]. By contrast, designing a CRISPR/Cas9 genome editing tool is exceedingly simple: it only involves modifying a 20 nucleotide stretch of the so-called guide (g)RNA component in the system such that it can bind a desired genomic DNA sequence via Watson-Crick basepairing, thereby directing the associated Cas9 to the target site to elicit a DSB $[5,15,16]$.

Adoptive $\mathrm{T}$ cell therapy is emerging as a new treatment modality for multiple types of cancers and has produced unprecedented promising results in the clinic $[17$, 18]. These approaches involve ex vivo manipulation of $\mathrm{T}$ cells to express either engineered $\mathrm{T}$-cell receptors (TCRs) or chimeric antigen receptors (CARs) capable of recognizing a particular tumour antigen, or they involve purification and expansion of tumour-infiltrating lymphocytes (TILs) [19]. Most T cell trials have so far used autologous (i.e. patient-derived) $\mathrm{T}$ cells. However, while the autologous strategy is simple from the viewpoint of immunogenicity and tolerance, there are substantial obstacles in terms of manufacturing time and expenses, as well as the poor quality and quantity of obtainable $\mathrm{T}$ cells, particularly for infants or heavily treated patients. For these reasons, allogeneic (i.e. donor-derived) $\mathrm{T}$ cell immunotherapies are currently being explored to circumvent the drawbacks of the autologous-based approach [20]. However, allogeneic $\mathrm{T}$ cell transfer also entails several challenges. One problem is that the endogenous TCR present on the infused allogeneic $\mathrm{T}$ cells may recognize antigens in the recipient beyond those present on the tumour cells, leading also to offtumor reactivity, termed graft-versus-host disease (GvHD). Such GvHD could generally be prevented by gene knockout of TCR in the donor T cells [21, 22]. TCR is composed of a TCR $\alpha$ chain, encoded by a single TRAC gene, complexed with a TCR $\beta$ chain, encoded by two TRBC genes. Since the TCR $\alpha \beta$ dimer is necessary for full function of TCR, its complete disruption can be achieved by knockout of TRAC [7]. Accordingly, the alloreactive potential of donor CAR $\mathrm{T}$ cells to elicit GvHD will be eliminated by knocking out their endogenous TRAC gene [23]. T cells lacking a TCR component lose CD3 expression and all capabilities for activation via either the CD3 complex or through the TCR. Another problem to be solved is the rejection of infused allogeneic $\mathrm{T}$ cells via host-versus-graft (HvG) reactions. It has been proposed that HvG reactions may be prevented by lymphodepleting regimens in the recipient [24], such as alkylating agents and purine nucleotide analogues compounds. Alternatively, lymphodepletion may be achieved using the anti-CD52 antibody alemtuzumab, when combined with knockout of the CD52 gene in the infused $\mathrm{T}$ cells, enabling them to evade the lymphodepletion regimen [23].

Given the importance of genomic engineering of human primary $\mathrm{T}$ cells for both basic research and immunotherapy, efficient and affordable systems to deliver the engineering reagents, now mainly CRISPR/ Cas9, to these cells are critical. Virus-mediated systems and plasmid electroporation are generally the most popular strategies for delivery. Compared with viralmediated delivery, electroporation of CRISPR/Cas9 expression plasmids is theoretically safer, because it does not entail integration of CRISPR/Cas9 into the genome. Moreover, this method is simpler, faster, and more economical relative to the viral-based delivery system [25].

In this study, we employed transfection of CRISPR/ Cas9 plasmid reagents for knockout of TRAC and CD52 genes. We demonstrate that transient expression of these reagents can knockout the TCR complex and CD52 in a human test cell line and in human primary $\mathrm{T}$ cells.

\section{Results}

\section{Generation of CRISPR/Cas9 reagents for knockout of TRAC and CD52}

As the first step towards engineering of human primary $\mathrm{T}$ cells, we designed gRNAs targeting exon one of either TRAC or CD52 and introduced them into pSpCas9-2A- 
GFP plasmid for dual expression of Cas9 and gRNA. We initially tested the ability of the gRNAs to elicit indels in $293 \mathrm{~T}$ cells, a cell line frequently used for validation of newly generated CRISPR/Cas9 reagents. Briefly, cells were transfected with gRNA-expressing pSpCas9-2AGFP plasmids. Three days after transfection, the cells were subjected to FACS to bulk isolate a cell population with high and homogenous gRNA/Cas9 expression, as revealed by the co-expressed GFP marker (population P4 in Fig. 1), which we previously showed translates into high editing levels [26]. Thereafter, genomic DNA was extracted from the FACS isolated cells and indel editing outcomes were evaluated by two different methods that analyse PCR products obtained by amplification of the genomic gRNA target site, i.e. TIDE and IDAA (see Materials and Methods for description of the methods).

For the CD52 gRNA, TIDE and IDAA revealed essentially the same indel types and extent of editing (Fig. 2 a \& b). A 1 bp insertion was the major indel in addition to several low-frequency deletions. Total and frame-shifting (knockout) indels were $\sim 89 \%$ and $\sim 83 \%$, respectively.
For the TRAC gRNA, the two indel characterization methods also revealed quite similar indel spectra, although a few of the indels were detected with significantly different frequencies. Overall, a $1 \mathrm{bp}$ insertion was a major indel, though less dominant (Fig. 2 a \& b). Total and frame-shifting indels were $\sim 65 \%$ and $\sim 62 \%$, respectively. Similar results were obtained in 3 independent experiments. Thus, both gRNAs were able to elicit a high degree of editing as well as knockout editing, as assessed in $293 \mathrm{~T}$ cells. Based on comparison with numerous other gRNAs, which we routinely screen in this system and under same conditions, the CD52 and $T R A C$ gRNAs can be categorized as very active.

\section{Functional inactivation of TCR or CD52 by CRISPR/Cas9 in human primary $\mathrm{T}$ cells}

Having validated that the gRNAs are functional, we proceeded to use them for functional inactivation of TCR and CD52 in activated (proliferating) human primary $\mathrm{T}$ cells. For delivery, we chose nucleofection, which is considered the most efficient delivery method of plasmids
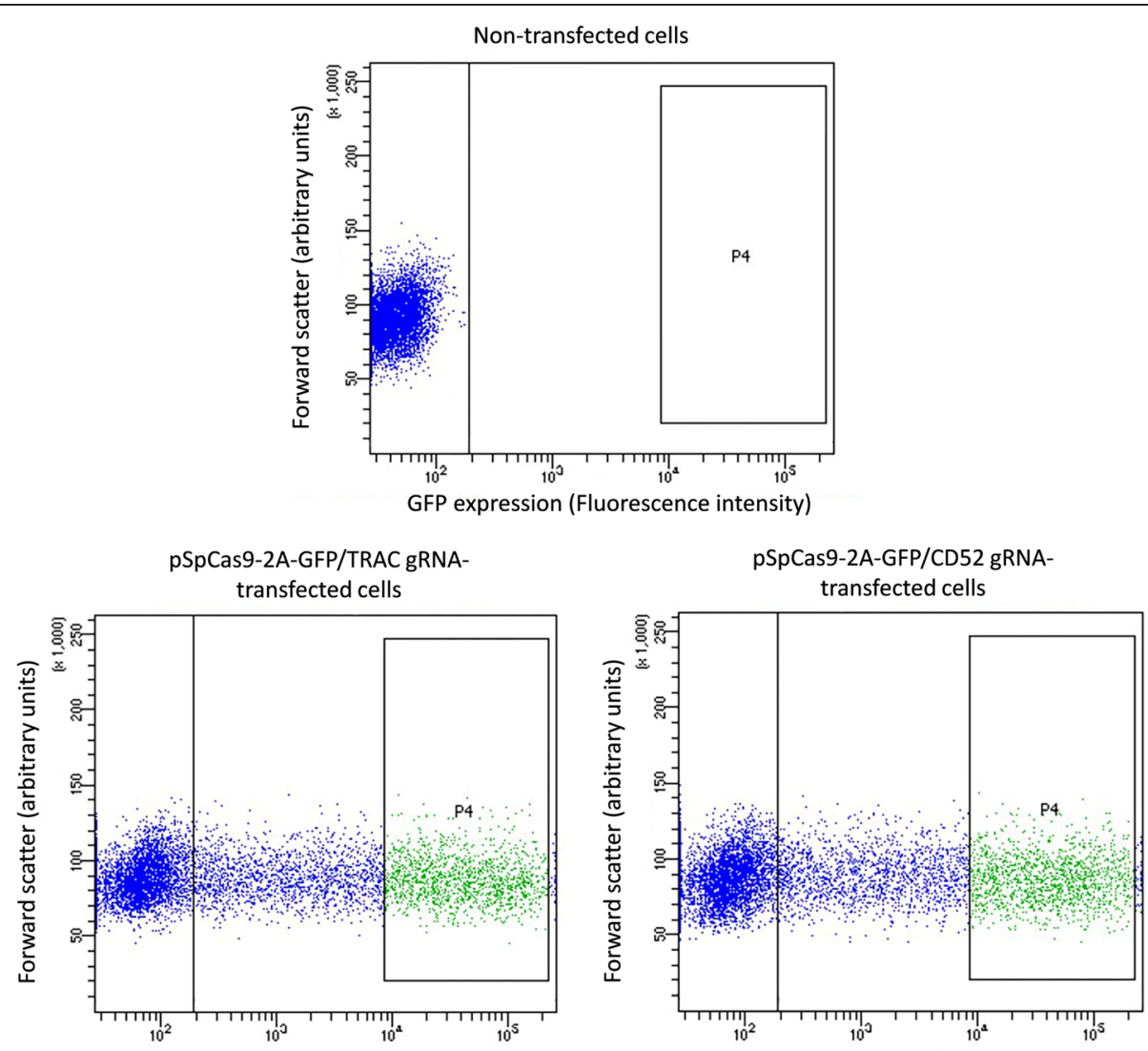

GFP expression (Fluorescence intensity)

GFP expression (Fluorescence intensity)

Fig. 1 FACS isolation of gRNA/Cas9-expressing 293 T cells. Three days after transfection of 293 T cells with no construct or TRAC or CD52 gRNAexpressing pSpCas9-2A-GFP construct, the cells were subjected to flow cytometry. Analysis of non-transfected cells was used to define the autofluorescence level of the cells in the GFP channel (marked by a bar in the graphs). Next, cells with high GFP expression levels (population 4) were bulk FACS isolated 


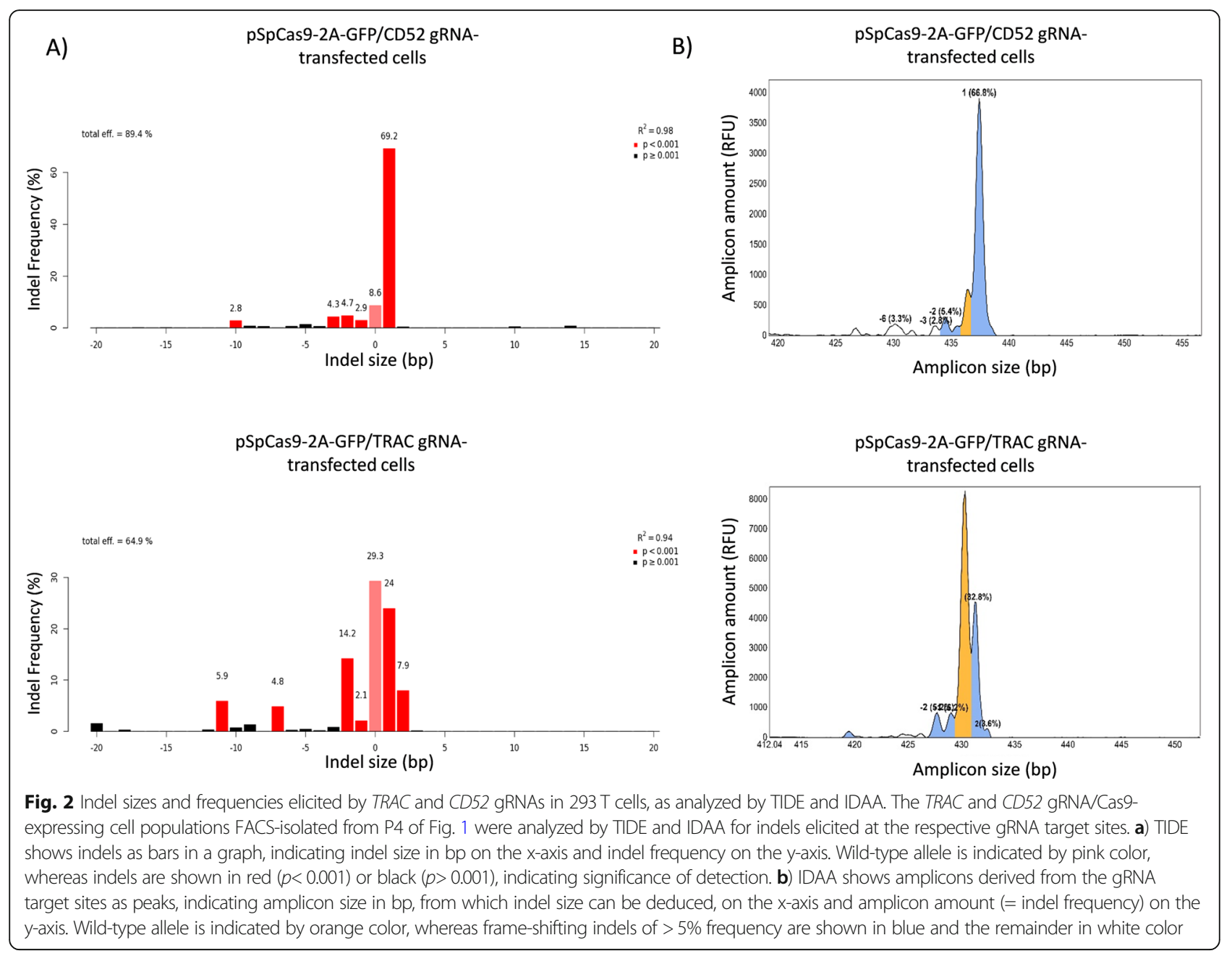

for these cells. Transfection efficiencies using various plasmid concentrations and electroporation pulse codes were assessed by nucleofection of pSpCas9-2A-GFP/ TRAC gRNA plasmid and flow cytometry quantification of GFP positive cells. The transfection efficiencies achieved under the most optimal conditions were around 24\% (Fig. 3) and these conditions were chosen for the subsequent experiments.

We next nucleofected activated $\mathrm{T}$ cells with either empty pSpCas9-2A-GFP/No gRNA plasmid or pSpCas9-2A-GFP plasmid expressing gRNA for either TRAC or CD52. Four days post-transfection, we purified genomic DNA and analysed the gRNA target sites by IDAA. Strikingly, in T cells, the mixed indel spectrum observed for both the TRAC and CD52 gRNAs in $293 \mathrm{~T}$ cells was replaced by one single 1-bp insertion (Fig. 4a). The mean frequencies and S.D. of this frameshifting indel were $14.3 \%+/-3 \%$ for the TRAC gRNA and $12.5 \%+/-1.4 \%$ for the CD52 gRNA, as determined in 3 experiments. Note that no indels at the TRAC or CD52 gRNA target sites were observed in cells transfected with empty pSpCas9-2AGFP/No gRNA plasmid, demonstrating that the editing was specific.

Finally, we quantified the cell surface levels of the targeted proteins on the edited T-cells by flow cytometry. To quantify TCR expression, we used a specific monoclonal antibody to monitor $\mathrm{CD} 3 \varepsilon$, which is only present on the $\mathrm{T}$ cell surface, when $\mathrm{TCR} \alpha \beta$ are expressed [27]. CD52 expression was quantified using the anti-CD52 monoclonal antibody, from which alemtuzumab is derived. As illustrated in Fig. 4b, flow cytometry revealed that the TRAC gRNA increased the fraction of cells with background levels of CD3 from $3 \%$ (Q1 in left panel) to $\sim 11 \%$ (Q5 in middle panel), suggesting functional knockout of TCR in $\sim 8 \%$ of the T cell population $(11-3 \%=8 \%)$. Similarly, the $C D 52$ gRNA increased the fraction of cells with CD52 back ground levels from $\sim 3 \%$ (Q3 in left panel) to $~ 11 \%(\mathrm{Q} 3$ in right panel), suggesting functional knockout of CD52 in 8\% of the $\mathrm{T}$ cell population $(11-3 \%=8 \%)$. The mean knockout frequencies and S.D. of similar quantifications were 

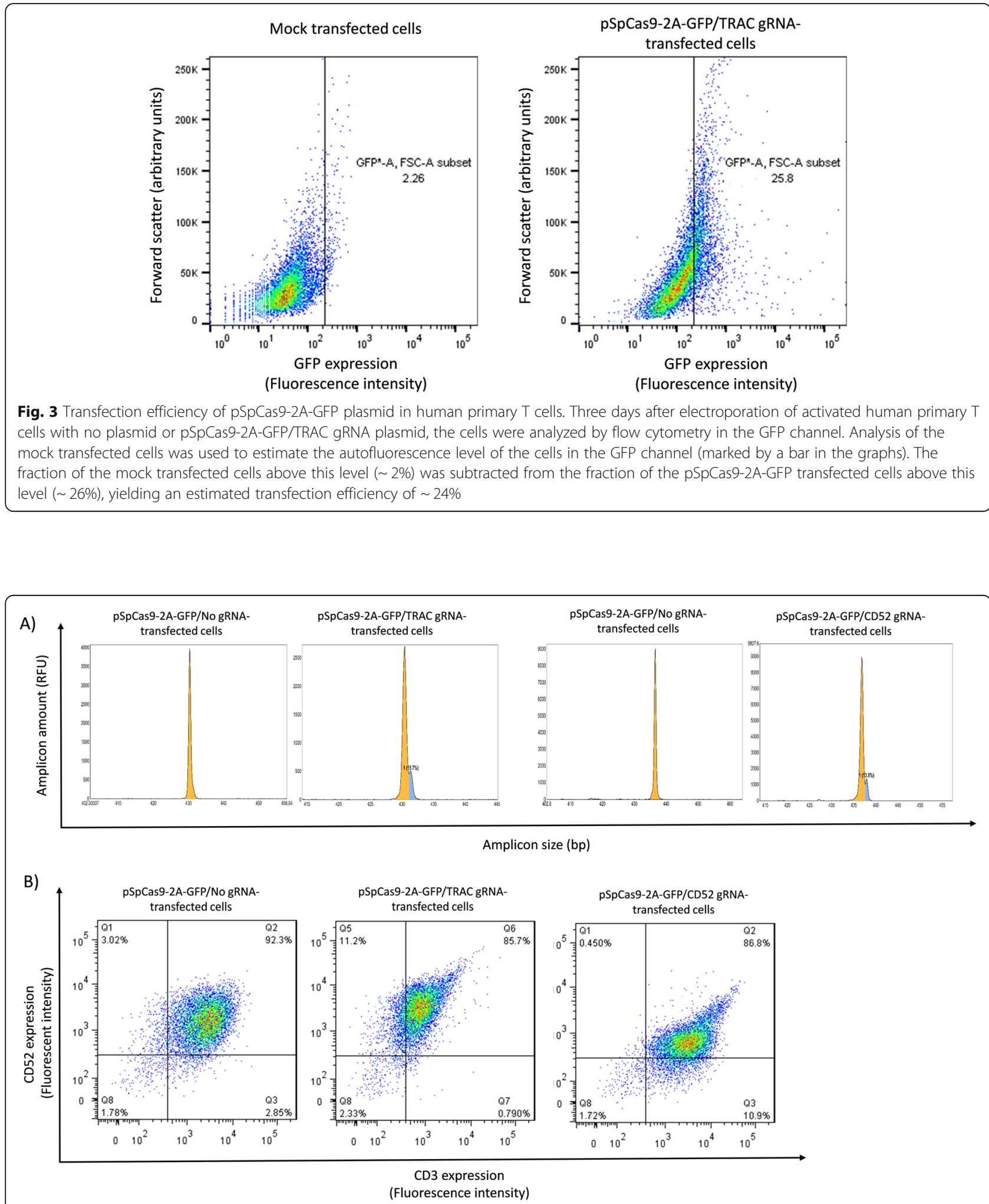

Fig. 4 Knockout of TRAC and CD52 in human primary T cells. Four days after transfection of T cells with empty (No gRNA) or TRAC or CD52 gRNAexpressing pSpCas9-2A-GFP construct, the cells were analysed by a) IDAA, revealing a 1-bp insertion elicited by both gRNAs and no indels in empty pSpCas9-2A-GFP/No gRNA transfected cells or by $\mathbf{b}$ ) flow cytometry for CD3 (TCR) and CD52 surface expression. This analysis showed an $\sim 8 \%$ increase of cells in the gates representing background CD3 or CD52 expression levels, indicating that TCR and CD52 knockout was achieved in $\sim 8 \%$ of the cells 
$7.1 \%+/-1 \%$ for the TRAC gRNA and $7.8 \%+/-0.3 \%$ for the CD52 gRNA, as determined in 3 experiments.

\section{Discussion}

In this study, we exploited the CRISPR/Cas9 gene editing platform to knockout two clinically relevant genes, TRAC and CD52, in human primary T cells. Our experimental workflow comprised isolation and activation of human primary $\mathrm{T}$ cells followed by electroporationbased transfer of plasmids expressing Cas9 and gRNAs specific for the TRAC and CD52 genes. Prior to testing in primary $\mathrm{T}$ cells, the activity of each gRNA was validated in the $293 \mathrm{~T}$ cell line.

The ability of both gRNAs to elicit indels (i.e. activity), as tested in $293 \mathrm{~T}$ cells could be extrapolated to primary $\mathrm{T}$ cells. However, the indel spectrum was different, as the bias towards a 1-bp insertion observed in $293 \mathrm{~T}$ cells was a complete predominance of the 1-bp insertion in $\mathrm{T}$ cells. One-bp insertions are elicited by the NHEJ pathway, which operates throughout the cell cycle, whereas indels $\geq 2 \mathrm{bp}$ are typically the result of MMEJ repair, which is restricted to S-phase and G2 of the cell cycle $[8,9,28,29]$. The observed shift therefore most likely reflects a more pronounced cell cycle arrest in the primary cells, as compared to the cell line. This may be due to the fact that the primary cells are more prone to p53mediated cell cycle arrest in response to the Cas9elicited DSB, as compared to the cell line [30] or that they are more sensitive to the toxic effects of the transfected plasmid DNA [31, 32]. Regardless of the mechanism, the data highlights that it is important to determine the indel spectrum of gRNAs for $\mathrm{T}$ cell editing in $\mathrm{T}$ cells, since indel spectra obtained in other cell types may be different. In the present case, our indel analyses demonstrated that both gRNAs elicited frame-shifting, i.e. functional knockout indels in human primary $\mathrm{T}$ cells. Accordingly, both gRNAs were also found to decrease TCR (CD3) and CD52 surface expression to background levels in $7-8 \%$ of the transfected $\mathrm{T}$ cells, which probably reflects knockout of both alleles in this cell population. A presumed, biallelic knockout of $7-8 \%$ is in good agreement with the observed $12-14 \%$ total frameshift indel mutagenesis, as biallelic:monoallelic indel modification often occur in 1:2-1:3 ratios in CRISPR/Cas9 editing.

To date, several attempts have been reported, which combine knockout of the endogenous TCR, using ZFNs $[2,21]$, TALENs $[22,23,33]$ or CRISPR/Cas9 $[7,34,35]$, with redirecting of $\mathrm{T}$ cells for cancer immunotherapy. In early efforts of generating allogeneic $\mathrm{T}$ cells, ZFNs and TALENs were used to inactivate the TRAC or TRBC genes [21, 22]. Both studies used mRNA electroporationbased protocols and achieved gene disruption rates of 20$30 \%$ for ZFNs [21] and $40-60 \%$ for TALENs [2]. In another study, electroporation of TALEN mRNAs were used to target TRAC and CD52 in CD19 CAR-T cells with disruption rates of $>70$ and $>60 \%$, respectively [23]. This study also demonstrated in vivo anti-tumor activity of the TCR/CD52-deficient CAR-T cells in a lymphoma murine model.

Now, CRISPR/Cas9 has become the genome editing technology of choice, and three major delivery systems have been developed: viral, plasmid and RNP. Early attempts to apply viral "all-in-one" CRISPR/Cas9 delivery systems in human primary $\mathrm{T}$ cells resulted in low targeting efficiencies [36, 37]. No attempts to increase the efficiencies have subsequently been reported, most likely due to the inherent risks associated with genomic integration of the editing tools. Plasmid based delivery has gained widespread use in the general editing community due to its many advantages and has therefore also been explored for $\mathrm{T}$ cell editing. The advantages include transient and therefore, in theory, safer delivery, ease of use, versatility, typically robust efficiencies and high stability as well as ease and affordability regarding production [38]. Moreover, thousands of CRISPR-related products are currently available as plasmids and a large knowledge base on plasmid-mediated CRISPR/Cas9 editing has been developed.

Unfortunately however, there is growing evidence that DNA-based approaches work inefficiently for CRISPR/ Cas9 gene editing in primary $\mathrm{T}$ cells $[34,39,40]$. In one report, gRNAs exhibiting substantial efficiency in $293 \mathrm{~T}$ cells showed low or no significant efficiency in primary $\mathrm{T}$ cells [31], in agreement with a subsequent study showing that high editing of plasmid-based CRISPR/Cas9 in cell lines may not translate into high editing activity in primary cells [40]. Our present work supports the notion of relatively inefficient editing of CRISPR/Cas9 plasmids in $\mathrm{T}$ cells. There may be several reasons for this, among which, relatively low transfection efficiencies represent one limiting factor, as also illustrated by our study. Thus, despite using nucleofection, which generally is the most efficient transfection approach for hard-to-transfect cells, we only achieved relatively modest delivery to the $\mathrm{T}$ cells. DNA toxicity is another likely obstacle for the use of plasmid-based CRISPR/Cas9 editing in $\mathrm{T}$ cells [31, 41], in part due to the innate immune response of $\mathrm{T}$ cells to double-stranded nucleic acids [42]. Looking forward, it is possible that the low efficiencies of CRISPR/ Cas 9 plasmids in T cells may be elevated by FACS-based genome editing: such approaches may enable isolation of a population of cells that all express CRISPR/Cas9, but at a defined and relatively low level that minimizes toxicity, yet allows sufficient editing, as has been described for other cell types [26].

The third, and more recently developed, RNP gene editing system is highly-priced, precluding exploitation 
by low-budget research groups, and the reagents are very fragile. However, RNPs seem to overcome the shortcomings described above for CRISPR/Cas9 plasmids: thus, they are appearing very efficient and do not pose the toxicity associated with plasmids [39, 43, 44]. Furthermore, they have high amenability to multiplex genome editing [43]. Altogether, RNPs are therefore emerging as the so far most promising method for CRISPR/Cas9based $\mathrm{T}$ cell.

\section{Conclusion}

In conclusion, our data demonstrate that genomic knockout using CRISPR/Cas9 plasmids expressing sgRNA and Cas9 is technically feasible in human primary $\mathrm{T}$ cells, however, at relatively low efficiencies, which will hamper its clinical application, where high efficiency will be desirable. Future efforts would need to solve the issues causing low efficiencies of CRISPR/Cas9 plasmids in $\mathrm{T}$ cells or alternatively, continue the development of the efficient RNP-based platform in primary $\mathrm{T}$ cells to provide a high-throughput method for therapeutic applications.

\section{Materials and methods}

\section{Guide RNA design and plasmid construction}

The gene sequences of human TRAC and CD52 genes were downloaded from NCBI and ensemble websites and gRNAs were designed to target the first exon of each gene using the CRISPR Design tool (http://crispr. mit.edu). For each gRNA, two complementary 5 '-phosphorylated oligonucleotides encompassing the gRNA sequence and BbsI restriction endonuclease site overhangs were synthesized (Table 1), annealed and sub-cloned into pSpCas9-2A-GFP plasmid (pX458, Addgene 48, 138\#, Cambridge, MA, USA) that had been digested with BbsI (New England Biolabs, Beverly, MA, USA), using a Golden Gate assembly cloning strategy [45], and gel purified using a Gel Extraction Kit (Qiagen). The resultant constructs were subjected to Sanger sequencing to verify proper sub-cloning of the gRNA sequences.

\section{Cell culture}

The human embryonic kidney (HEK) $293 \mathrm{~T}$ cell line (ATCC, cat. no. CRL-11268) was maintained in

Table 1 Oligos used for introduction of gRNA sequences for TRAC and CD52 genes into pSpCas9-2A-GFP plasmid

\begin{tabular}{ll}
\hline Name & Sequence \\
\hline TRAC-gRNA (F/top) & 5'p-CACCGTCTCTCAGCTGGTACACGGC-3' \\
TRAC-gRNA (R/bottom) & 5'p-AAACGCCGTGTACCAGCTGAGAGAC-3' \\
CD52-gRNA (F/top) & 5'p-CACCGCAGCCTCCTGGTTATGGTAC-3' \\
CD52-gRNA (R/bottom) & 5'p-AAACGTACCATAACCAGGAGGCTGC-3' \\
\hline The gRNA sequences are underlined
\end{tabular}

Dulbecco's Modified Eagle's Medium (high glucose). Peripheral blood mononuclear cells were isolated from blood (buffy coats) of healthy volunteer donor (following written informed consent) using Ficoll-Paque density gradient. $\mathrm{CD}^{+} \mathrm{T}$ cells were isolated from peripheral blood mononuclear cells using the Pan T Cell Isolation Kit (Miltenyi Biotec, Bergisch Gladbach, Germany) according to the manufacturer's guidelines. $\mathrm{T}$ cells were expanded through activation by anti-human CD3/CD28 magnetic dynabeads (Thermo Fisher Scientific, Inc., Waltham, MA, USA) at a bead to cell ratio of $1: 1$ and $30 \mathrm{IU} / \mathrm{mL}$ of recombinant human IL-2 (Miltenyi Biotec, Bergisch Gladbach, Germany) in RPMI 1640 medium (Gibco; Thermo Fisher Scientific, Inc., Waltham, MA, USA) supplemented with Glutamax (Gibco; Thermo Fisher Scientific, Inc., Waltham, MA, USA) and $1 \mathrm{mM}$ sodium pyruvate. All media were supplemented with 0.1 $\mathrm{mg} / \mathrm{mL}$ Penicillin and Streptomycin (Gibco; Thermo Fisher Scientific, Inc., Waltham, MA, USA), and 10\% Fetal Bovine Serum (Gibco; Thermo Fisher Scientific, Inc., Waltham, MA, USA). All cells were cultured at $37^{\circ} \mathrm{C}$ in humidified atmosphere with $5 \% \mathrm{CO} 2$.

\section{T cell transfection}

Plasmids were introduced into $\sim 80 \%$ confluent $293 \mathrm{~T}$ cells in 24-well $\left(2 \mathrm{~cm}^{2}\right)$ plates using Lipofectamine ${ }^{\mathrm{ma}} 3000$ (Life Technologies, San Diego, CA, USA) according to the manufacturer's instructions. Briefly, $0.5 \mu \mathrm{g}$ of gRNAexpressing pSpCas9(BB)-2A-GFP plasmid construct was diluted in $25 \mu \mathrm{L}$ Opti-MEM reduced serum media (Gibco; Thermo Fisher Scientific, Inc., Waltham, MA, USA) supplemented with $1 \mu \mathrm{L}$ of P3000 Reagent (Life Technologies). Then, $1.5 \mu$ l Lipofectamine 3000 reagent was diluted in $25 \mu \mathrm{L}$ Opti-MEM and thereafter mixed with the diluted DNA/P3000 Reagent. The mixtures were incubated at room temperature for $5 \mathrm{~min}$ and thereafter added dropwise to each well of cells.

\section{Primary $\mathrm{T}$ cell transfection}

Three days after activation of the $\mathrm{T}$ cells, the $\mathrm{CD} 3 / \mathrm{CD} 28$ Dynabeads were magnetically removed and $\mathrm{T}$ cells were cultured in the absence of beads for $6-12 \mathrm{~h}$ and thereafter electroporated using the P3 Primary Cell 4DNucleofector $^{\mathrm{ru}}$ X Kit S, (V4XP-3032, Lonza, Cologne, Germany), according to the following protocol: briefly, $1 \times 10^{6}$ cells were washed twice with phosphate-buffered saline by centrifuging at $300 \mathrm{~g}$ for $5 \mathrm{~min}$ and thereafter resuspended together with the gRNA-expressing pSpCas9-2A-GFP plasmid in nucleofection buffer. The resulting cell mixture was transferred to nucleofection cuvette and immediately subjected to nucleofection in an Amaxa 4D-Nucleofection device, using program EH115. After nucleofection, the cell mixture was gently 
transferred to a dish with pre-warmed medium and incubated at $37^{\circ} \mathrm{C}$.

\section{Fluorescence activated cell sorting (FACS) of GFP- expressing $293 \mathrm{~T}$ cells}

Three days after transfection, $293 \mathrm{~T}$ cells were subjected to FACS isolation of cells with high levels of GFP, encoded by pSpCas9-2A-GFP, and consequently high levels of editing, essentially, using procedures we described previously [26]. Briefly, the cells were detached using trypsin, then passed through a $50-\mu \mathrm{m}$ filcon (BD Biosciences, San Jose, CA) to achieve a single-cell suspension and finally sorted for a desired GFP fluorescence level in a BD FACS Aria $^{\text {Ta }}$ III instrument (BD Biosciences, San Jose, CA), using non-transfected cells to define the background fluorescence level. After FACS isolation, cells were harvested.

\section{Evaluation of indel mutagenesis}

The CRISPR/Cas9-induced indel frequencies were quantified by Indel Detection by Amplicon Analysis (IDAA), using procedures we described previously [26] and by Tracking of Indels by Decomposition (TIDE), as described by others [46]. IDAA is based on PCR amplification of the gRNA target site of the edited sample using a tri-primer amplicon fluorescence labeling set-up that allows determination of amplicon sizes and frequencies by subsequent DNA fragment analysis and quantification by capillary electrophoresis in a sequenator [26]. Amplicons containing a deletion or an insertion will be shorter or longer than amplicons derived from the wild-type allele, thereby allowing determination of indel sizes, and the relative indel frequencies are reflected by peak size. TIDE analysis is based on two PCR amplifications of the gRNA target site, performed on the edited sample and a wild-type (unedited) control sample. The PCR products are next subjected to standard Sanger sequencing and the two sequence data files are uploaded to the TIDE webtool (http://tide.nki.nl). The sequencing traces are then analyzed using a specially developed algorithm, which is provided as an easy-to-use web tool that determines the indels present in the edited sample and quantifies frequencies by computational decomposition of the mixture of sequence traces in the edited sample relative to the control sample.

Briefly, genomic DNA was first extracted from cells using QuickExtract buffer (Epicentre, Madison, WI, USA), yielding lysates of $3000-4000$ cells/ $\mu$ l that were incubated at $65^{\circ} \mathrm{C}$ for $20 \mathrm{~min}$, followed by $98^{\circ} \mathrm{C}$ for 10 min. For IDAA, the tri-primer set-up encompassed: a gene-specific forward primer with a common $5^{\prime}$ overhang, a gene-specific reverse primer, and a universal 6FAM 5'-labelled forward primer (FamFwd) which has same sequence as the overhang on the gene-specific
Table 2 IDAA primers

\begin{tabular}{ll}
\hline Primer name & Sequence (5' to $\mathbf{3}^{\prime}$ ) \\
\hline IDAA-CD52-Fwd & 5'-AGCTGACCGGCAGCAAAATTGTGACGAT \\
IDAA-CD52-Rev & CTAGCCTGCTCC-3' \\
& 5'-AGTCCATCTGCTGTGCTCTC-3' \\
IDAA-TRAC-Fwd & 5'-AGCTGACCGGCAGCAAAATTGTCACGAG \\
IDAA-TRAC-Rev & CAGCTGGTTTCTA-3' \\
& 5'-ATGCTGTTGTGAAGGCGTT-3' \\
\hline
\end{tabular}

forward primer (Table 2). PCR amplification was done using TEMPase Hot Start DNA Polymerase (Amplicon, Denmark), 10:1:10 ratio of FamFwd:Fwd:Rev primers and touchdown thermocycling conditions: an initial $72^{\circ} \mathrm{C}$ annealing temperature ramping down by 1 degree/ cycle to $58^{\circ} \mathrm{C}$, followed by an additional 25 cycles using $58^{\circ} \mathrm{C}$ annealing temperature. Dilutions of the PCR products were mixed with GS500LIZ size standard (Thermo Fisher Scientific) and applied to fragment analysis on an ABI3500 sequenator (ABI/Life Technologies, USA) according to the manufacturer's instructions. Data were analyzed using Viking ProfileIt ${ }^{\mathrm{tm}}$ indel profiling software (https://viking.sdu.dk).

For TIDE, purified PCR products amplifying the gRNA target sites from edited and wild-type samples were Sanger-sequenced and analyzed with the TIDE webtool (http://tide.nki.nl).

\section{Flow Cytometry}

TCR and CD52 surface disruption was quantified using flow cytometry analysis 4 days post-electroporation. Transfected primary $\mathrm{T}$ cells were thoroughly washed with phosphate-buffered saline containing 1\% Fetal Bovine Serum and stained with mouse APC-conjugated anti-human CD3 (BD Biosciences) or PE-conjugated anti-human CD52 (BD Biosciences) for 20-30 min at $4{ }^{\circ} \mathrm{C}$ in dark. As isotype controls were used mouse APCconjugated IgG1א (BD Biosciences) or mouse PEconjugated IgG3k (BD Biosciences). A BD FACSCalibur ${ }^{\text {Tx }}$ instrument (BD Bioscience) was used to perform the flow cytometry analysis. All data were analyzed using FlowJo Software.

\begin{abstract}
Abbreviations
CRISPR: clustered regularly-interspaced short palindromic repeats; TCR: T cell receptor; CAR: chimeric antigen receptor; GVHD: graft-versus-host-disease; ZFN: zinc-finger nucleases; TALEN: transcription activator-like effector nuclease; NHEJ: non-homologous end joining; MMEJ: microhomology-mediated end-joining; TIL: tumour-infiltrating lymphocytes; DSB: double-strand break; InDel: insertion or deletion; HvG: host-versus-graft; IDAA: Indel Detection by Amplicon Analysis; TIDE: Tracking of Indels by Decomposition; HEK: human embryonic kidney; FACS: Fluorescence Activated Cell Sorting
\end{abstract}

\section{Acknowledgements}

Not applicable.

\section{Authors' contributions}

$F R$, EK, ZH, and MF contributed to the conception and design of the study. $M F$, FR, and ZH supervised the study. EK coordinated the project and 
performed the experiments underlying the manuscript. EK and MF analyzed and interpreted the results. EK wrote the original draft of the manuscript. MF assisted in writing and editing of the manuscript. The author(s) read and approved the final version of manuscript.

\section{Funding}

This study was funded by the Graduate Office of the University of Isfahan (Grant No. 8178) and National Institute for Medical Research Development (Grant No. 958883). The funders had no roles in the study design, collection, analysis, and interpretation of data and in writing of the manuscript.

\section{Availability of data and materials}

The data that support the findings of this study are available from the corresponding authors upon reasonable request.

\section{Ethics approval and consent to participate}

The research involving use of human specimen complied with the relevant national regulations, institutional policies, in accordance with the Helsinki Declaration and have been approved by the research ethics committee of University of Isfahan. Blood samples were collected from healthy volunteers following written informed consent.

\section{Consent for publication}

Not applicable.

\section{Competing interests}

The authors declare that they have no competing interests.

\section{Author details}

${ }^{1}$ Department of Cell and Molecular Biology \& Microbiology, Faculty of Biological Science and Technology, University of Isfahan, Isfahan, Iran. ${ }^{2}$ Department of Medical Biotechnology, Faculty of Medical Sciences, Tarbiat Modares University, Tehran, Iran. ${ }^{3}$ Biotech Research and Innovation Centre (BRIC), Faculty of Health Sciences, University of Copenhagen, Copenhagen, Denmark.

Received: 5 September 2020 Accepted: 22 December 2020

Published online: 29 January 2021

\section{References}

1. Khan SH. Genome-editing technologies: concept, pros, and cons of various genome-editing techniques and bioethical concerns for clinical application. Molecular therapy Nucleic acids. 2019;16:326.

2. Provasi E, Genovese P, Lombardo A, Magnani Z, Liu P-Q, Reik A, et al. Editing $T$ cell specificity towards leukemia by zinc finger nucleases and lentiviral gene transfer. Nat Med. 2012;18(5):807.

3. Boissel S, Jarjour J, Astrakhan A, Adey A, Gouble A, Duchateau P, et al. megaTALs: a rare-cleaving nuclease architecture for therapeutic genome engineering. Nucleic Acids Res. 2013;42(4):2591-601.

4. Cermak T, Doyle EL, Christian M, Wang L, Zhang Y, Schmidt C, et al. Efficient design and assembly of custom TALEN and other TAL effector-based constructs for DNA targeting. Nucleic acids research. 2011;39(12):e82-e.

5. Cong L, Ran FA, Cox D, Lin S, Barretto R, Habib N, et al. Multiplex genome engineering using CRISPR/Cas systems. Science. 2013;1231143.

6. Urnov FD, Rebar EJ, Holmes MC, Zhang HS, Gregory PD. Genome editing with engineered zinc finger nucleases. Nat Rev Genet. 2010;11(9):636.

7. Osborn MJ, Webber BR, Knipping F, Lonetree C-L, Tennis N, AP DF, et al, Evaluation of TCR gene editing achieved by TALENs, CRISPR/Cas9, and megaTAL nucleases. Mol Ther. 2016:24(3):570-81.

8. Ceccaldi R, Rondinelli B, D'Andrea AD. Repair pathway choices and consequences at the double-strand break. Trends Cell Biol. 2016:26(1):52-64.

9. Chang HH, Pannunzio NR, Adachi N, Lieber MR. Non-homologous DNA end joining and alternative pathways to double-strand break repair. Nat Rev Mol Cell Biol. 2017:18(8):495.

10. Deriano L, Roth DB. Modernizing the nonhomologous end-joining repertoire: alternative and classical NHEJ share the stage. Annu Rev Genet. 2013;47:433-55.

11. Hustedt N, Durocher D. The control of DNA repair by the cell cycle. Nat Cell Biol. 2017;19(1):1-9.

12. Bennett EP, Petersen BL, Johansen IE, Niu Y, Yang Z, Chamberlain CA, et al. INDEL detection, the 'Achilles heel'of precise genome editing: a survey of methods for accurate profiling of gene editing induced indels. Nucleic Acids Res. 2020;48(21):11958-81.

13. McVey M, Lee SE. MMEJ repair of double-strand breaks (director's cut) deleted sequences and alternative endings. Trends Genet. 2008:24(11): 529-38.

14. Silva G, Poirot L, Galetto R, Smith J, Montoya G, Duchateau P, et al. Meganucleases and other tools for targeted genome engineering: perspectives and challenges for gene therapy. Current gene therapy. 2011; 11(1):11-27.

15. Mali P, Yang L, Esvelt KM, Aach J, Guell M, DiCarlo JE, et al. RNA-guided human genome engineering via Cas9. Science. 2013;339(6121):823-6.

16. Gaj T, Gersbach CA, Barbas CF III. ZFN, TALEN, and CRISPR/Cas-based methods for genome engineering. Trends Biotechnol. 2013;31(7):397-405.

17. Khalil DN, Smith EL, Brentjens RJ, Wolchok JD. The future of cancer treatment: immunomodulation, CARs and combination immunotherapy. Nat Rev Clin Oncol. 2016;13(5):273.

18. Morris EC, Stauss HJ. Optimizing T-cell receptor gene therapy for hematologic malignancies. Blood, The Journal of the American Society of Hematology. 2016;127(26):3305-11.

19. Maus MV, Fraietta JA, Levine BL, Kalos M, Zhao Y, June CH. Adoptive immunotherapy for cancer or viruses. Annu Rev Immunol. 2014;32:189-225.

20. Wang $X$, Rivière I. Clinical manufacturing of CAR T cells: foundation of a promising therapy. Molecular Therapy-Oncolytics. 2016;3.

21. Torikai H, Reik A, Liu P-Q, Zhou Y, Zhang L, Maiti S, et al. A foundation for" universal" T-cell based immunotherapy: T-cells engineered to express a CD19-specific chimeric-antigen-receptor and eliminate expression of endogenous TCR. Blood. 2012:blood-2012-01-405365.

22. Berdien B, Mock U, Atanackovic D, Fehse B. TALEN-mediated editing of endogenous $\mathrm{T}$-cell receptors facilitates efficient reprogramming of $\mathrm{T}$ lymphocytes by lentiviral gene transfer. Gene Ther. 2014;21(6):539.

23. Poirot L, Philip B, Schiffer-Mannioui C, Le Clerre D, Chion-Sotinel I, Derniame $\mathrm{S}$, et al. Multiplex genome edited T-cell manufacturing platform for "off-the-shelf" adoptive T-cell immunotherapies. Cancer research. 2015:canres. 3321.2014.

24. Muranski P, Boni A, Wrzesinski C, Citrin DE, Rosenberg SA, Childs R, et al Increased intensity lymphodepletion and adoptive immunotherapy-how far can we go? Nat Rev Clin Oncol. 2006;3(12):668

25. Zhang Z, Qiu S, Zhang X, Chen W. Optimized DNA electroporation for primary human T cell engineering. BMC Biotechnol. 2018;18(1):4.

26. Lonowski LA, Narimatsu Y, Riaz A, Delay CE, Yang Z, Niola F, et al. Genome editing using FACS enrichment of nuclease-expressing cells and indel detection by amplicon analysis. Nat Protoc. 2017;12(3):581-603.

27. Geisler C, Kuhlmann J, Rubin B. Assembly, intracellular processing, and expression at the cell surface of the human alpha beta T cell receptor/ CD3 complex. Function of the CD3-zeta chain. J Immunol. 1989;143(12): 4069-77.

28. Allen F, Crepaldi L, Alsinet C, Strong AJ, Kleshchevnikov V, De Angeli $P$, et al. Predicting the mutations generated by repair of Cas9-induced doublestrand breaks. Nat Biotechnol. 2019;37(1):64-72

29. Taheri-Ghahfarokhi A, Taylor BJM, Nitsch R, Lundin A, Cavallo A-L, MadeyskiBengtson $\mathrm{K}$, et al. Decoding non-random mutational signatures at Cas9 targeted sites. Nucleic Acids Res. 2018;46(16):8417-34.

30. Haapaniemi E, Botla S, Persson J, Schmierer B, Taipale J. CRISPR-Cas9 genome editing induces a p53-mediated DNA damage response. Nat Med. 2018:24(7):927-30

31. Mandal PK, Ferreira LM, Collins R, Meissner TB, Boutwell CL, Friesen M, et al. Efficient ablation of genes in human hematopoietic stem and effector cells using CRISPR/Cas9. Cell Stem Cell. 2014;15(5):643-52.

32. Knipping F, Osborn MJ, Petri K, Tolar J, Glimm H, von Kalle C, et al. Genomewide specificity of highly efficient TALENs and CRISPR/Cas9 for T cell receptor modification. Molecular Therapy-Methods \& Clinical Development. 2017:4:213-24.

33. Qasim W, Zhan H, Samarasinghe S, Adams S, Amrolia P, Stafford S, et al. Molecular remission of infant B-ALL after infusion of universal TALEN geneedited CAR T cells. Science translational medicine. 2017;9(374):eaaj2013.

34. Ren J, Zhang X, Liu X, Fang C, Jiang S, June $\mathrm{CH}$, et al. A versatile system for rapid multiplex genome-edited CAR T cell generation. Oncotarget. 2017; 8(10):17002

35. Morton LT, Reijmers RM, Wouters AK, Kweekel C, Remst DF, Pothast CR, et al. Simultaneous Deletion of Endogenous TCRaß for TCR Gene Therapy Creates an Improved and Safe Cellular Therapeutic. Molecular Therapy. 2019. 
36. Wang W, Ye C, Liu J, Zhang D, Kimata JT, Zhou P. CCR5 gene disruption via lentiviral vectors expressing Cas9 and single guided RNA renders cells resistant to HIV-1 infection. PLoS One. 2014;9(12):e115987.

37. Li C, Guan X, Du T, Jin W, Wu B, Liu Y, et al. Inhibition of HIV-1 infection of primary CD4+ T-cells by gene editing of CCR5 using adenovirus-delivered CRISPR/Cas9. J Gen Virol. 2015;96(8):2381-93.

38. Chicaybam L, Sodre AL, Curzio BA, Bonamino MH. An efficient low cost method for gene transfer to T lymphocytes. PLoS One. 2013:8(3):e60298.

39. Schumann K, Lin S, Boyer E, Simeonov DR, Subramaniam M, Gate RE, et al. Generation of knock-in primary human T cells using Cas9 ribonucleoproteins. Proc Natl Acad Sci. 2015;112(33):10437-42.

40. Hendel A, Bak RO, Clark JT, Kennedy AB, Ryan DE, Roy S, et al. Chemically modified guide RNAs enhance CRISPR-Cas genome editing in human primary cells. Nature biotechnology. 2015;33(9):nbt. 3290.

41. Su S, Hu B, Shao J, Shen B, Du J, Du Y, et al. CRISPR-Cas9 mediated efficient PD-1 disruption on human primary $T$ cells from cancer patients. Sci Rep. 2016;6:20070

42. Monroe KM, Yang Z, Johnson JR, Geng X, Doitsh G, Krogan NJ, et al. IFI16 DNA sensor is required for death of lymphoid CD4 T cells abortively infected with HIV. Science. 2013;1243640.

43. Liang X, Potter J, Kumar S, Zou Y, Quintanilla R, Sridharan M, et al. Rapid and highly efficient mammalian cell engineering via Cas9 protein transfection. J Biotechnol. 2015;208:44-53.

44. Gundry MC, Brunetti L, Lin A, Mayle AE, Kitano A, Wagner D, et al. Highly efficient genome editing of murine and human hematopoietic progenitor cells by CRISPR/Cas9. Cell Rep. 2016;17(5):1453-61.

45. Engler C, Kandzia R, Marillonnet S. A one pot, one step, precision cloning method with high throughput capability. PLoS One. 2008;3(11):e3647.

46. Brinkman EK, Chen T, Amendola M, van Steensel B. Easy quantitative assessment of genome editing by sequence trace decomposition. Nucleic acids research. 2014;42(22):e168-e.

\section{Publisher's Note}

Springer Nature remains neutral with regard to jurisdictional claims in published maps and institutional affiliations.

Ready to submit your research? Choose BMC and benefit from:

- fast, convenient online submission

- thorough peer review by experienced researchers in your field

- rapid publication on acceptance

- support for research data, including large and complex data types

- gold Open Access which fosters wider collaboration and increased citations

- maximum visibility for your research: over $100 \mathrm{M}$ website views per year

At $\mathrm{BMC}$, research is always in progress.

Learn more biomedcentral.com/submissions 